\title{
Incidental finding of small bowel perforation by a foreign body
}

\author{
Tanveer Amin, Ghali Midhat, Jayasuriya Neil
}

\begin{abstract}
Introduction: Generally in adults, most foreign body ingestion occurs accidentally, but may be as a result of contributory factors such as psychiatric disorders, mental retardation, alcohol consumption, and an edentulous state. The ingested foreign bodies usually pass uneventfully through the gastrointestinal tract within one week. Perforation occurs in less than 1\% of all patients. Patients may present with vague abdominal pain with no known history of foreign body ingestion. Case Series: Herein, we report two cases of bowel perforations by ingesting foreign body who did not recall the ingestion that required surgical intervention. Conclusion: Clinicians should suspect such condition in the presence of some predisposing factors, and a surgical consultation is necessary.
\end{abstract}

Keywords: Abdominal pain, Bowel perforation, Ingestion of foreign body (IFB)

\section{How to cite this article}

Amin T, Midhat G, Neil J. Incidental finding of small bowel perforation by a foreign body. Case Rep Int 2016;5:31-35.

Tanveer Amin ${ }^{1}$, Ghali Midhat ${ }^{1}$, Jayasuriya Neil ${ }^{1}$ Affiliations: ${ }^{1}$ General Surgical Department, Latrobe Regional Health Services, Traralgon, Victoria, Australia. Corresponding Author: Amin Tanveer, General Surgical Department, Latrobe Regional Health Services, Traralgon, Victoria, Australia; E-mail: amintanveer@hotmail.com

Received: 28 May 2016

Accepted: 19 July 2016

Published: 03 August 2016
Article ID: 100027 CRINTTA2016

$* * * * * * * * *$

doi:10.5348/crint-2016-27-CS-8

\section{INTRODUCTION}

In adults, most foreign body ingestion occurs accidentally, but may be as a result of contributory factors such as psychiatric disorders, mental retardation, alcohol consumption, and an edentulous state. Generally; patients do not recall ingesting a foreign body and this is usually detected on radiological imaging studies, during surgery or in the pathological examination of the surgical specimens.

Hereby, we are presenting two interesting cases:

The first case was admitted for elective laparoscopic anterior resection of a diverticular mass and repair of colovesical fistula, found to have a small bowel perforation by a sharp pointed chicken bone detected by the CT scan in the postoperative period.

The second case presented with abdominal pain to our emergency department, found to have small bowel perforation by a bamboo stick.

These case reports intend to draw attention towards possibility of silent foreign body ingestion in patients presenting with vague abdominal pain with no known history of foreign body ingestion, clinician should suspect such conditions in the presence of some predisposing factors and a surgical consultation is usually necessary.

\section{CASE SERIES}

\section{Case 1}

A 63-year-old male was admitted for elective laparoscopic anterior resection of sigmoid diverticular 
mass and repair of colovesical fistula on the background of chronic abdominal pain. His past medical and surgical history included chronic obstructive airway disease, ischemic heart disease, hypertension, appendectomy, cholecystectomy and recurrent sigmoid diverticulitis. He was a retired chef and had top dentures. He had an uncomplicated procedure. On postoperative day-10 his Jackson drain was removed.

On postoperative day-12 the patient had a plain abdominal X-ray and CT scan for investigation and assessment of prolonged postoperative paralytic ileus.

The abdominal X-ray was reported normal, however, the CT scan was reported query remains of drain tube tip inside the abdominal cavity (Figure 1 and Figure 2), and a small incisional hernia at the port site.

This case was discussed in a multidisciplinary meeting, and a decision was made to remove the foreign body and repair the incisional hernia at the same time.

Initially, it was a laparoscopic approach but after being unable to locate the foreign body it was converted to an open midline laparotomy.

The small bowel was traced through its entire course, a chicken bone was found sticking out of the jejunum posteriorly with the jejunal loop adherent to the posterior abdominal wall with formation a fistula. The chicken bone (Figure 3) was removed and the jejunum was primarily repaired with interrupted sutures, followed by repair of the incisional hernia. His postoperative recovery was complicated by anemia, hospital acquired pneumonia, and electrolyte imbalances. He was discharged in a good clinical condition.

\section{Case 2}

A 32-year-old male presented to emergency department with four day history of central abdominal pain, associated with nausea. His past surgical history included appendicectomy and vasectomy. On examination, his vital signs were stable; on abdominal examination he had generalized abdominal tenderness mostly central with no signs of peritonism. His blood tests result revealed hemoglobin 149, WCC 12.3 Neut 9.0, and CRP 137. Blood gases, urea, creatinine, liver function tests, and lipase were unremarkable. A plain abdominal $\mathrm{X}$-ray was reported normal, however, a focal area of inflammatory change surrounding a loop of ileum was detected on the CT scan which was reported most likely to be a Meckel's diverticulitis (Figure 4).

On the basis of imaging findings it was decided to perform an emergency surgical intervention. Initially a laparoscopic approach was performed, during the procedure an inflammatory knot of mid small bowel with a localized distal ileal perforation from a fistulating bamboo stick was detected (Figures 5 and 6), also the small bowel was tethered on the mesentery. Therefore, the procedure was converted to a midline laparotomy. A wedge excision of the small bowel and a primary end to end anastomosis were performed. Postoperative recovery of this patient was complicated by a pulmonary embolism. He was transferred to a tertiary hospital for management of the pulmonary embolism. Eventually, he was discharged in a good condition.

\section{DISCUSSION}

Swallowing of foreign bodies is most common in children aged between six months and six years $[1,2]$.

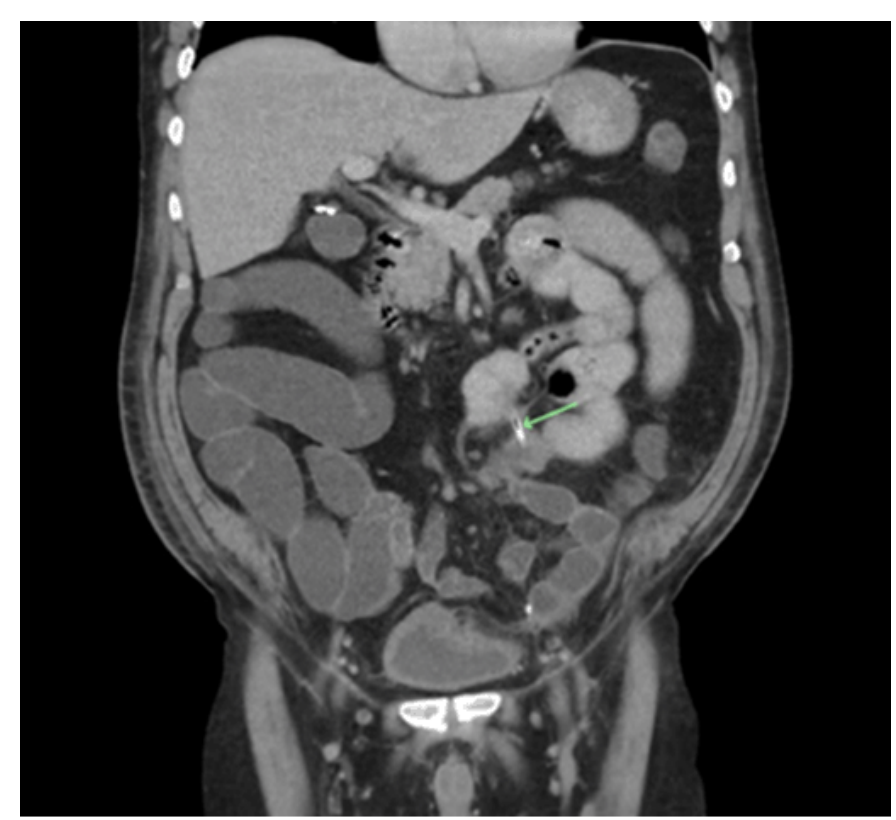

Figure 1: The arrow showing the foreign body.

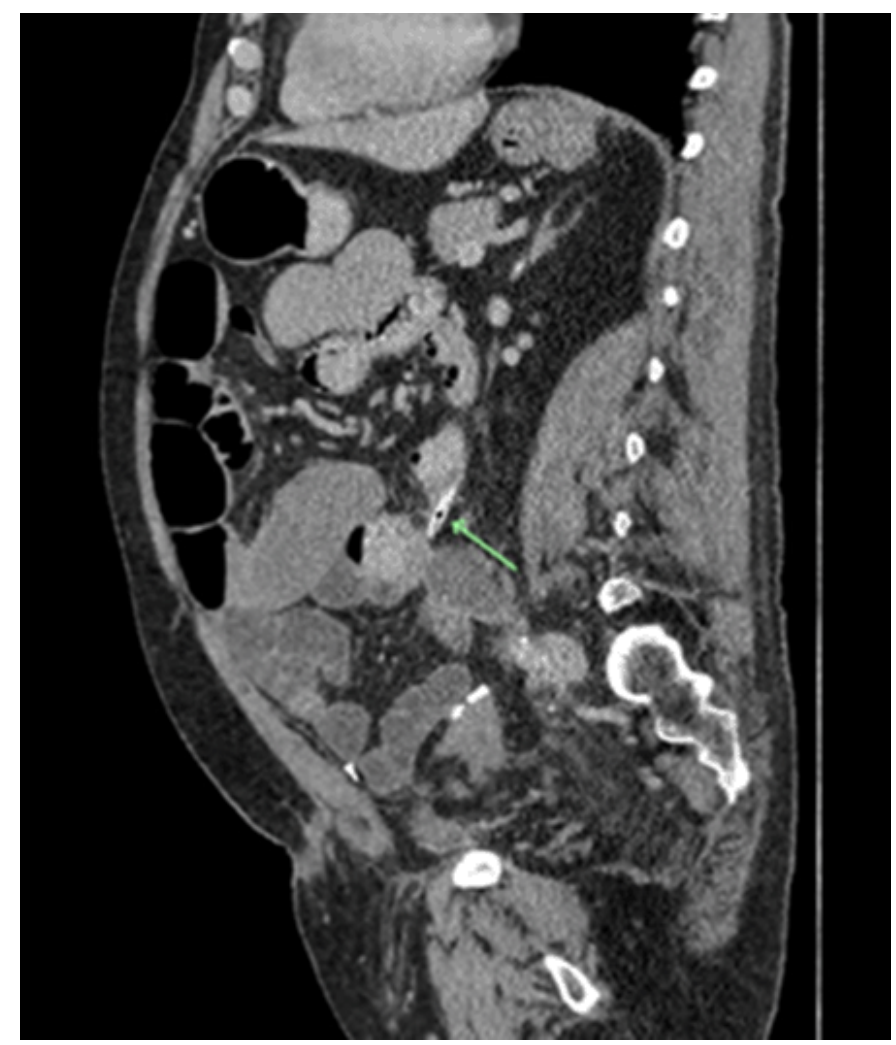

Figure 2: The arrow showing the foreign body. 
In adults, ingestion of foreign body are usually seen in patients with psychiatric disorders, mental retardation, alcohol consumption, and an edentulous state. Elderly people may have trouble using dentures and as the sense of feeling in the palate is decreased, they may become prone to foreign body ingestion. Generally, patients do not recall ingesting a foreign body and this is usually detected on radiological imaging studies, during surgery or in the pathological examination of the surgical specimens [3]. In these two cases the patient did not recall the incidence.

Ingestion of foreign body usually presents with nonspecific symptoms. Abdominal pain is the most common complaint (95\%), followed by fever (81\%) and localized

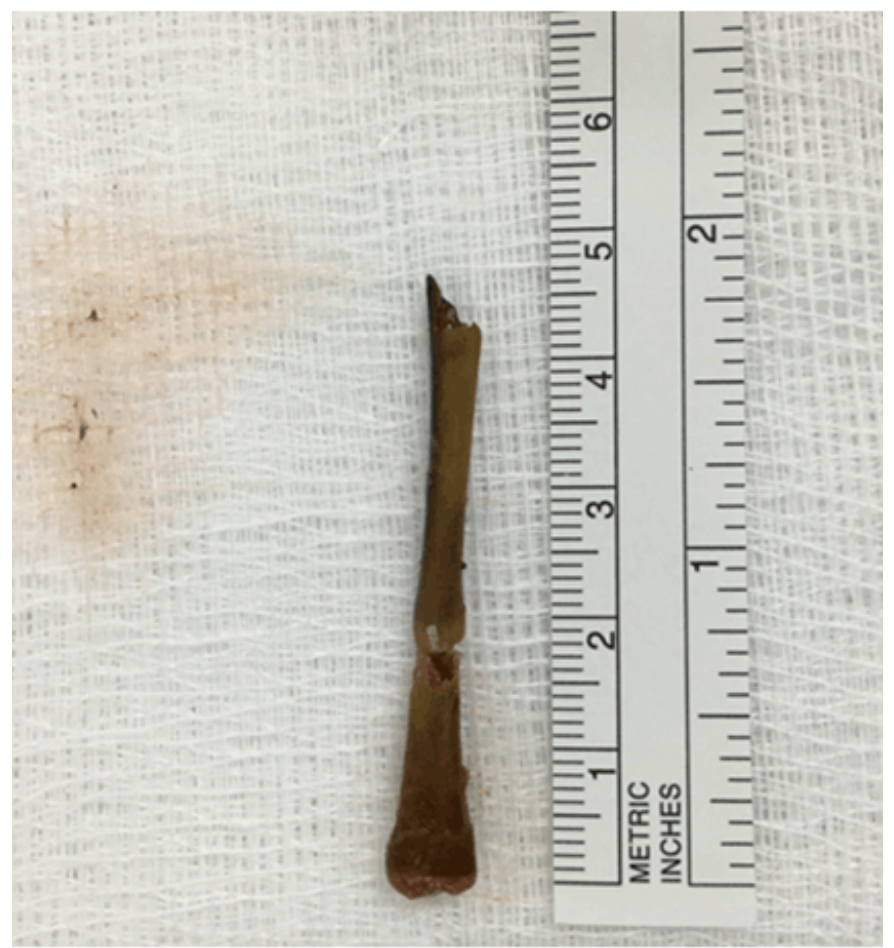

Figure 3: Sharp pointed chicken bone.

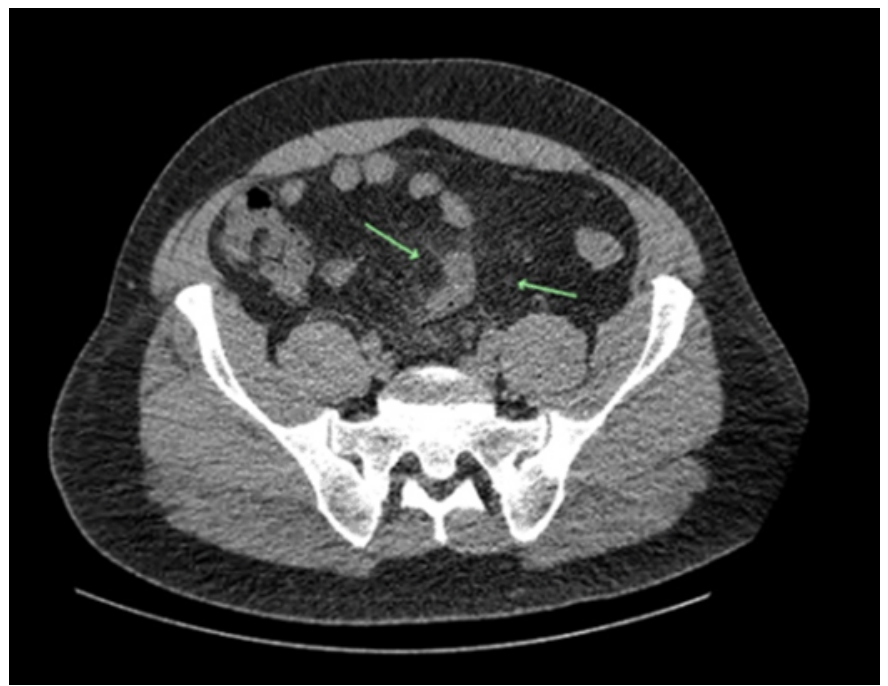

Figure 4: An inflammatory change surrounding a loop of ileum. peritonitis (39\%). The other symptoms that may occur are nausea, vomiting, hematochezia and melena. Preoperative diagnosis remains a challenge and therefore it must be considered in susceptible cases with presence of some predisposing factors [4].

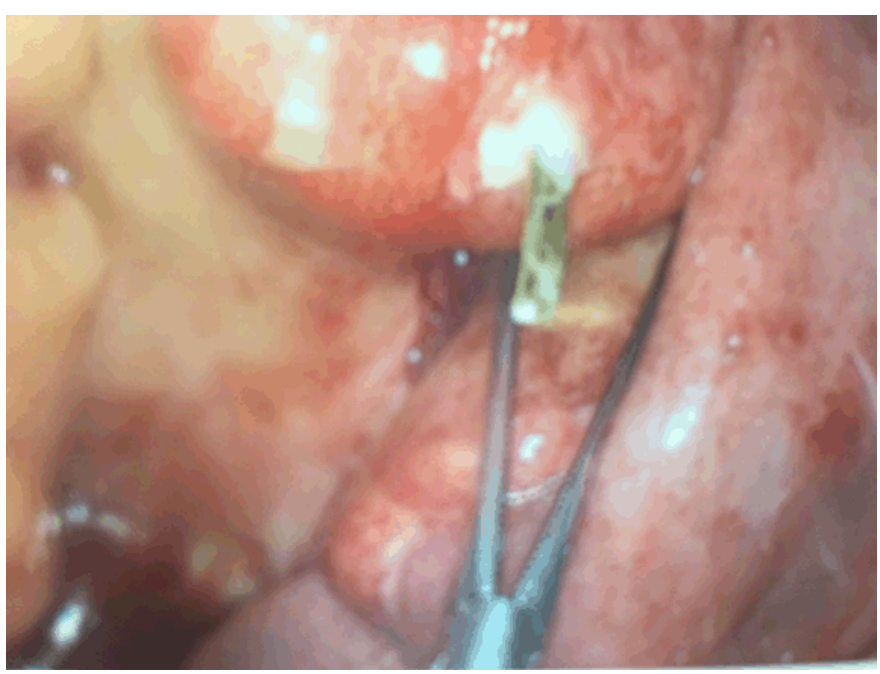

Figure 5: Sharp bamboo stick perforating ileum.

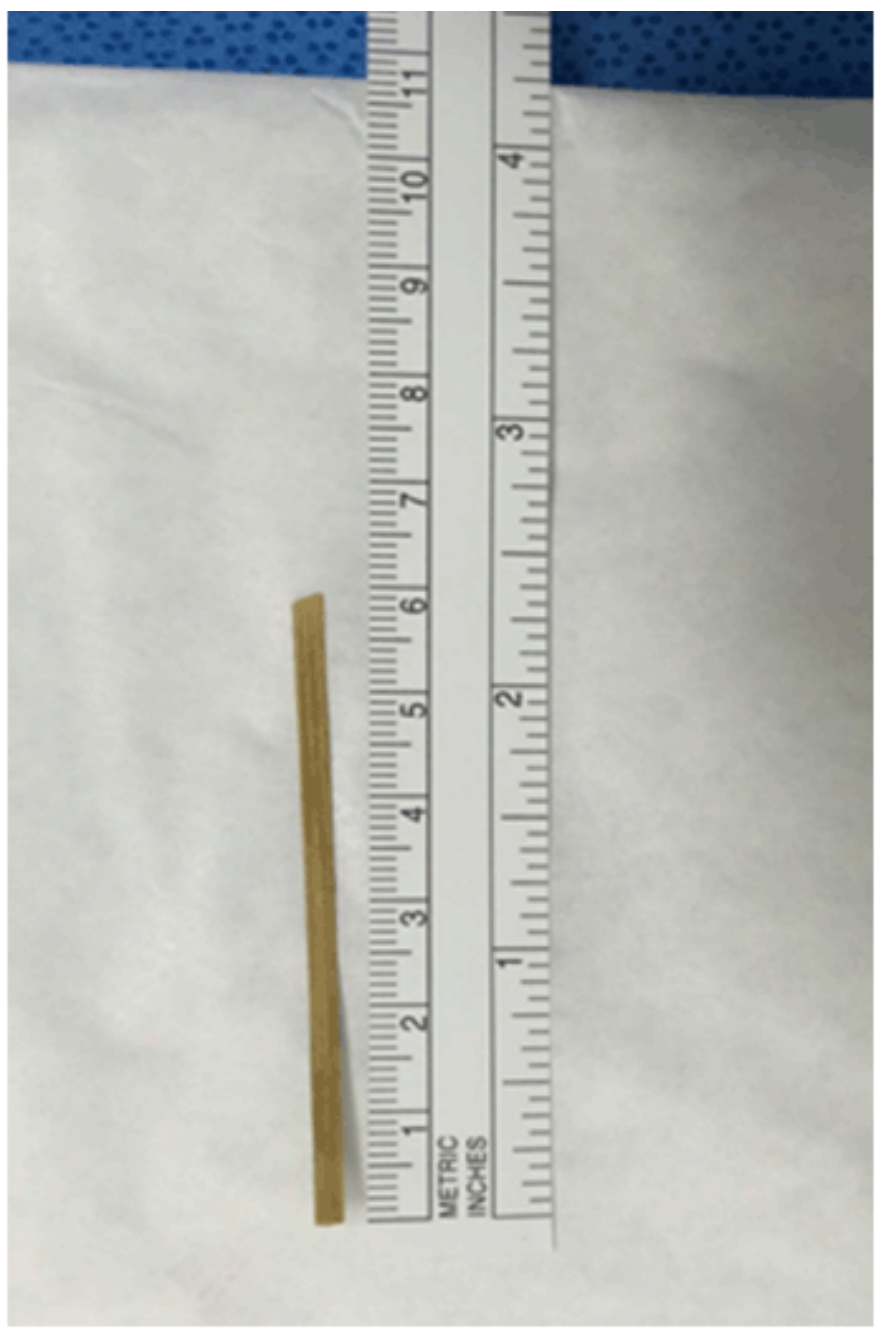

Figure 6: Sharp bamboo stick. 
The most common foreign bodies are food stuffs or their parts, such as fish bones, bone fragments or vegetable bezoars and toothpicks [5]. Although generally the ingested bones are digested or uneventfully pass through the gastrointestinal tract within one week, complications such as impaction, perforation or obstruction may rarely occur [6-8].

Gastrointestinal perforation occurs in less than $1 \%$ of all patients. The possibility of perforation is associated with the length and sharpness of the swallowed object [68]. Previous bowel disease such as, history of abdominal or bowel operation, inflammatory bowel disease, bowel tumors, diverticular disease, abdominal wall hernias and a blind loop of bowel increase the risk of perforation. Ingested sharp bones, fish and chicken bones can lead to intestinal perforation and peritonitis. Goh et al. [10] state that most of the foreign bodies causing gastrointestinal tract perforation were of a food origin, such as fish bones, chicken bones, bone fragments or shells.

Bowel perforations can occur at any part of the intestinal tract, the most common sites are pylorus, ileocecal region and rectosigmoid junction [9]. Goh et al. [10] reported terminal ileum perforation in $38.6 \%$ cases. Yilmaz et al. reported jejunal perforation is less frequent approximately $14.3 \%$.

Foreign bodies such as fish bones and chicken bones are dense enough to show in a plain X-ray, but most of the time is unidentified as concealed by fluids and soft tissue mass. These can be further investigated by using computed tomography (CT) scan. As Courier et al. [9] show the role of CT scan in the evaluation of igestion of foreign body, with sensitivity of $100 \%$ and a specificity of $91 \%$. In our cases, cCase 1 patient abdominal X-ray and Ccase 2 abdominal CT scan re-evaluated by the radiologist after the operation, was unable to identify the foreign body.

Absolute indication for surgery includes perforation and obstruction. Surgery is also indicated in complications that cannot resolve endoscopically or unsuccessful endoscopic attempts or in cases the ingested foreign bodies have remained in the same place for longer than a week.

The appropriate surgical intervention is depending according to the pathological finding and anatomic location of perforation, as laparoscopy is less invasive compare to laparotomy, it is the first option.

\section{CONCLUSION}

Silent foreign body ingestion in patients presenting with vague abdominal pain with no known history of foreign body ingestion is not uncommon. As reported, in our both cases patients did not recall ingestion of foreign body; Therefore it must be considered in susceptible cases. Clinicians should suspect such conditions in the presence of some predisposing factors, and a surgical consultation is usually necessary.

\section{$* * * * * * * * *$}

\section{Acknowledgements}

We acknowledge Dr Anusha Naidoo (Radiologist) for reevaluations of the patients imaging and Dr Simon Bazaadut for his contributions.

\section{Author Contributions}

Tanveer Amin - Substantial contributions to conception and design, Acquisition of data, Analysis and interpretation of data, Drafting the article, Revising it critically for important intellectual content, Final approval of the version to be published

Ghali Midhat - Analysis and interpretation of data, Revising it critically for important intellectual content, Final approval of the version to be published

Jayasuriya Neil - Analysis and interpretation of data, Revising it critically for important intellectual content, Final approval of the version to be published

\section{Guarantor}

The corresponding author is the guarantor of submission.

\section{Conflict of Interest}

Authors declare no conflict of interest.

\section{Copyright}

(C) 2016 Tanveer Amin et al. This article is distributed under the terms of Creative Commons Attribution License which permits unrestricted use, distribution and reproduction in any medium provided the original author(s) and original publisher are properly credited. Please see the copyright policy on the journal website for more information.

\section{REFERENCES}

1. Webb WA. Management of foreign bodies of the upper gastrointestinal tract: update. Gastrointest Endosc 1995 Jan;41(1):39-51.

2. Cheng W, Tam PK. Foreign-body ingestion in children: experience with 1,265 cases. J Pediatr Surg 1999 Oct;34(10):1472-6.

3. Yilmaz M, Akbulut S, Ozdemir F, Gozeneli O, Baskiran A, Yilmaz S. A swallowed dental prosthesis causing duodenal obstruction in a patient with schizophrenia: Description of a new technique. Int J Surg Case Rep 2012;3(7):308-10.

4. Lubel J, Wiley M. Gastrointestinal: Foreign bodies and diverticulitis. J Gastroenterol Hepatol 2005 Apr;20(4):649.

5. Rodríguez-Hermosa JI, Codina-Cazador A, Sirvent JM, Martín A, Gironès J, Garsot E. Surgically treated perforations of the gastrointestinal tract caused by ingested foreign bodies. Colorectal Dis 2008 Sep;10(7):701-7.

6. Akhtar S, McElvanna N, Gardiner KR, Irwin ST. Bowel perforation caused by swallowed chicken bones--a case series. Ulster Med J 2007 Jan;76(1):37-8. 
10. Goh BK, Tan YM, Lin SE, et al. CT in the preoperative diagnosis of fish bone perforation of the gastrointestinal tract. AJR Am J Roentgenol 2006 Sep;187(3):710-4.

11. Chin EH, Hazzan D, Herron DM, Salky B. Laparoscopic retrieval of intraabdominal foreign bodies. Surg Endosc 2007 Aug;21(8):1457.

12. Emir S, Ozkan Z, Altinsoy HB, Yazar FM, Sözen S, Bali I. Ingested bone fragment in the bowel: Two cases and a review of the literature. World J Clin Cases 2013 Oct $16 ; 1(7): 212-6$.
Access full text article on other devices

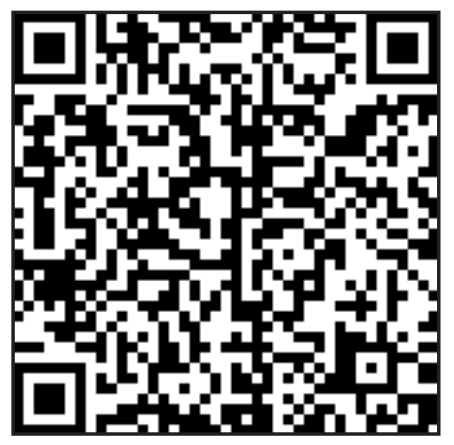

Access PDF of article on other devices

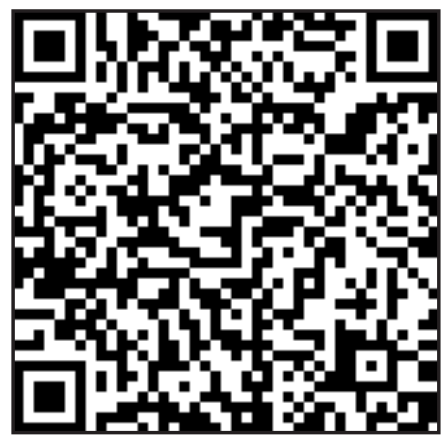

